\title{
The Relaxation Limits of the Two-Fluid Compressible Euler-Maxwell Equations
}

\author{
WANG Shu and ZHENG Lin* \\ College of Applied Science, Beijing University of Technology, Beijing 100124, China.
}

Received 26 February 2017; Accepted 31 October 2017

\begin{abstract}
In this paper we consider the relaxation limits of the two-fluid Euler-Maxwell systems with initial layer. We construct an asymptotic expansion with initial layer functions and prove the convergence between the exact solutions and the approximate solutions.
\end{abstract}

AMS Subject Classifications: 35Q35, 76W05

Chinese Library Classifications: $\mathrm{O} 193$

Key Words: Euler-Maxwell equation; relaxation limit; initial layer; asymptotic expansion.

\section{Introduction}

In this paper, we consider the three-dimensional two-fluid (including electrons and ions) Euler-Maxwell equations in a torus $\mathbb{T}=(\mathbb{R} / \mathbb{Z})^{3}$ :

$$
\begin{aligned}
& \partial_{t} n_{\alpha}+\operatorname{div}\left(n_{\alpha} u_{\alpha}\right)=0, \\
& m_{\alpha}\left[\partial_{t}\left(n_{\alpha} u_{\alpha}\right)+\operatorname{div}\left(n_{\alpha} u_{\alpha} \otimes u_{\alpha}\right)\right]+\nabla p\left(n_{\alpha}\right)=q_{\alpha} n_{\alpha}\left(E+u_{\alpha} \times B\right)-\frac{m_{\alpha} n_{\alpha} u_{\alpha}}{\tau_{\alpha}}, \\
& \varepsilon \partial_{t} E-\frac{1}{\mu} \nabla \times B=n_{e} u_{e}-n_{i} u_{i}, \\
& \partial_{t} B+\nabla \times E=0, \\
& \varepsilon \operatorname{div} E=n_{i}-n_{e}, \quad \operatorname{div} B=0,
\end{aligned}
$$

where $\alpha=e, i, q_{i}=1, q_{e}=-1 ; n_{e}$ and $n_{i}$ stand for the density of the electrons and ions; $u_{e}$ and $u_{i}$ stand for the velocity of the electrons and ions; $E$ and $B$ are respectively the electric

\footnotetext{
*Corresponding author. Email address: zhenglin0103@emails.bjut.edu.cn (L. Zheng), wangshu@bjut.edu.cn (S. Wang) 
field and magnetic field; $p=p\left(n_{\alpha}\right)$ is the pressure function which is sufficiently smooth and strictly increasing for $n_{\alpha}>0$. These variables are functions of a three-dimensional position vector $x \in \mathbb{T}$ and of the time $t>0$. In the above systems the physical parameters are the electron mass $m_{e}$ and the ion mass $m_{i}$, the momentum relaxation times $\tau_{e}$ and $\tau_{i}$, and the permittivity $\varepsilon$ and the permeability $\mu$.

For simplicity, we denote $m_{\alpha}=1, \varepsilon, \mu=1$ and $\tau_{e}=\tau_{i}=\tau$, then we obtain the following systems:

$$
\begin{aligned}
& \partial_{t} n_{\alpha}+\operatorname{div}\left(n_{\alpha} u_{\alpha}\right)=0, \\
& \partial_{t}\left(n_{\alpha} u_{\alpha}\right)+\operatorname{div}\left(n_{\alpha} u_{\alpha} \otimes u_{\alpha}\right)+\nabla p\left(n_{\alpha}\right)=q_{\alpha} n_{\alpha}\left(E+u_{\alpha} \times B\right)-\frac{n_{\alpha} u_{\alpha}}{\tau_{\alpha}}, \\
& \partial_{t} E-\nabla \times B=n_{e} u_{e}-n_{i} u_{i}, \quad \operatorname{div} E=n_{i}-n_{e}, \\
& \partial_{t} B+\nabla \times E=0, \quad \operatorname{div} B=0 .
\end{aligned}
$$

Furthermore, we make the time scaling by replacing $t$ by $\frac{t}{\tau}$ and define the enthalpy function $h\left(n_{\alpha}\right)$ by

$$
h\left(n_{\alpha}\right)=\int_{1}^{n_{\alpha}} \frac{p^{\prime}(s)}{s} \mathrm{~d} s .
$$

So the system we considered is rewritten the following reduced two-fluid EulerMaxwell systems:

$$
\begin{aligned}
& \partial_{t} n_{\alpha}+\frac{1}{\tau} \operatorname{div}\left(n_{\alpha} u_{\alpha}\right)=0, \\
& \partial_{t} u_{\alpha}+\frac{1}{\tau}\left(u_{\alpha} \cdot \nabla\right) u_{\alpha}+\frac{1}{\tau} \nabla h\left(n_{\alpha}\right)=\frac{q_{\alpha}\left(E+u_{\alpha} \times B\right)}{\tau}-\frac{u_{\alpha}}{\tau^{2}}, \\
& \partial_{t} E-\frac{1}{\tau} \nabla \times B=\frac{n_{e} u_{e}-n_{i} u_{i}}{\tau}, \quad \operatorname{div} E=n_{i}-n_{e}, \\
& \partial_{t} B+\frac{1}{\tau} \nabla \times E=0, \quad \operatorname{div} B=0,
\end{aligned}
$$

with initial data:

$$
\left.\left(n_{\alpha}, u_{\alpha}, E, B\right)\right|_{t=0}=\left(n_{\alpha, 0}^{\tau}, u_{\alpha, 0}^{\tau}, E_{0}^{\tau}, B_{0}^{\tau}\right) .
$$

The study of compressible Euler-Maxwell equations began in 2000, Chen, Jerome and Wang [1] prove the existence of global weak solutions of the simplified Euler-Maxwell equations by using the method of step by step Godunov scheme combined with compensated compactness; in 2007 and 2008, Peng and Wang [2,3] study the non relativistic limit convergence problem for compressible Euler-Maxwell equations to compressible EulerPoisson equations and the composite limits of the quasi neutral limit and the non relativistic limit for compressible Euler-Maxwell equations; Peng, Wang and Gu [4] discuss the relaxation limit of compressible Euler-Maxwell equations and the existence of global smooth solution in 2011; in the same year, Wang, Yang and Zhao [5] research the relaxation limit of the plasma two-fluid Euler-Maxwell equations with the help of Maxwell 
iteration and energy method for the well-prepared initial data; Mohamed-Lasmer Hajjej and Peng [6] study the relaxation limits of the one-fluid Euler-Maxwell equation with initial layer by the method of asymptotic expansion.

For later use in this paper, we recall some inequalities in Sobolev spaces $[7,8]$ and the local existence of smooth solutions for symmetrizablle hyperbolic equation. For any $s>0$, we denote by $\|\cdot\|_{s}$ the norm of the usual Sobolev space $H^{s}(\mathbb{T})$, and by $\|\cdot\|$ and $\|\cdot\|_{\infty}$ the norms of $L^{2}(\mathbb{T})$ and $L^{\infty}(\mathbb{T})$, respectively.

Lemma 1.1. (See $[9,10])$ Let $s \geq 3$ be an integer and $\left(n_{\alpha, 0}^{\tau}, u_{\alpha, 0}^{\tau}, E_{0}^{\tau}, B_{0}^{\tau}\right) \in H^{s}(\mathbb{T})$ with $n_{\alpha, 0}^{\tau} \geq k$ for some given constant $k>0$, independent of $\tau$. Then there exist $T_{1}^{\tau}>0$ and a unique smooth solution $\left(n_{\alpha}^{\tau}, u_{\alpha}^{\tau}, E^{\tau}, B^{\tau}\right)$ to the periodic problem (1.6)-(1.9) defined in the time interval $\left[0, T_{1}^{\tau}\right]$, with $\left(n_{\alpha}^{\tau}, u_{\alpha}^{\tau}, E^{\tau}, B^{\tau}\right) \in C^{1}\left(\left[0, T_{1}^{\tau}\right] ; H^{s-1}(\mathbb{T})\right) \cap C\left(\left[0, T_{1}^{\tau}\right] ; H^{s}(\mathbb{T})\right)$.

The main result is as follows:

Theorem 1.1. For any integer $s \geq 3$, under the Proposition 1.1 and the following conditions:

$$
\begin{aligned}
& \operatorname{div} E_{\tau}=n_{i, \tau}-n_{e, \tau}, \quad \operatorname{div} B_{\tau}=0, \\
& \sup _{0 \leq t \leq T_{1}}\left\|\left(n_{\alpha, \tau}, E_{\tau}, B_{\tau}\right)(t, \cdot)\right\|_{s} \leq C, \quad \sup _{0 \leq t \leq T_{1}} \|\left(u_{\alpha, \tau}(t, \cdot) \|_{s} \leq C,\right. \\
& \|\left(n_{\alpha, 0}^{\tau}-n_{\alpha, \tau}(0, \cdot), u_{\alpha, 0}^{\tau}-u_{\alpha, \tau}(0, \cdot), E_{0}^{\tau}-E_{\tau}(0, \cdot), B_{0}^{\tau}-B_{\tau}(0, \cdot) \|_{s} \leq C \tau^{2},\right.
\end{aligned}
$$

here $\left(n_{\alpha, \tau}, u_{\alpha, \tau}, E_{\tau}, B_{\tau}\right)(t, x)$ is the approximate solutions of (1.11)-(1.14), such that as $\tau \rightarrow 0$ we have $T_{1}^{\tau} \geq T_{1}$ and the solution $\left(n_{\alpha}^{\tau}, u_{\alpha}^{\tau}, E^{\tau}, B^{\tau}\right)$ satisfies:

$$
\begin{aligned}
& \left\|\left(n_{\alpha}^{\tau}, u_{\alpha}^{\tau}, E^{\tau}, B^{\tau}\right)-\left(n_{\alpha, \tau}, u_{\alpha, \tau}, E_{\tau}, B_{\tau}\right)\right\|_{s} \leq C \tau^{2}, \\
& \left\|u_{\alpha}^{\tau}-u_{\alpha, \tau}\right\|_{L^{2}\left(0, T_{\tau} ; H^{s}\right)} \leq C \tau^{3} .
\end{aligned}
$$

for any $t \in\left[0, T^{\tau}\right]$, here $T^{\tau} \leq T_{1}$.

Our main purpose in this paper is to study the relaxation limits of the two-fluid EulerMaxwell systems (1.11)-(1.14) with initial data (1.15). We consider the problem with initial layer. In order to establish our result, we make an asymptotic expansion including initial layer. In Section 2, we will take the approximate expression into systems (1.11)(1.14) such that we get the error estimates of the remainders. In Section 3, we prove the main result about the convergence.

\section{Asymptotic expansion}

In this section, we make an asymptotic expansion with initial layer functions and take it into the systems, then we can get the expression of the first-order initial layer functions and second-order initial layer functions. Furthermore, we obtain the estimates of the remainders which produced by approximate solutions and extra solutions. 
We know that

$$
\left(n_{\alpha, \tau}, u_{\alpha, \tau}, E_{\tau}, B_{\tau}\right)=\left(\tilde{n}_{\alpha, \tau}, \tilde{u}_{\alpha, \tau}, \tilde{E}_{\tau}, \tilde{B}_{\tau}\right)+\left(n_{\alpha, I}, u_{\alpha, I}, E_{I}, B_{I}\right),
$$

where $\left(n_{\alpha, \tau}, u_{\alpha, \tau}, E_{\tau}, B_{\tau}\right)$ is the approximate solution, $\left(\tilde{n}_{\alpha, \tau}, \tilde{u}_{\alpha, \tau}, \tilde{E}_{\tau}, \tilde{B}_{\tau}\right)$ the inner function, and $\left(n_{\alpha, I}, u_{\alpha, I}, E_{I}, B_{I}\right)$ is the initial layer function.

Firstly, we make the following ansatz for inner function

$$
\left(\tilde{n}_{\alpha, \tau}, \tilde{u}_{\alpha, \tau}, \tilde{E}_{\tau}, \tilde{B}_{\tau}\right)(t, x)=\sum_{j>0} \tau^{2 j}\left(n_{\alpha}^{j}, \tau u_{\alpha}^{j}, E^{j}, \tau B^{j}\right),
$$

and take it into systems $(1.11)-(1.14)$, we obtain

$$
\begin{aligned}
& \partial_{t} n_{\alpha}^{0}+\operatorname{div}\left(n_{\alpha}^{0} u_{\alpha}^{0}\right)=0, \\
& \nabla h\left(n_{\alpha}^{0}\right)=q_{\alpha} E^{0}-u_{\alpha}^{0}, \\
& \partial_{t} E^{0}-\nabla \times B^{0}=\frac{n_{e}^{0} u_{e}^{0}-n_{i}^{0} u_{i}^{0}}{\tau}, \quad \operatorname{div} E^{0}=n_{i}^{0}-n_{e}^{0}, \\
& \nabla \times E^{0}=0, \quad \operatorname{div} B^{0}=0,
\end{aligned}
$$

there $\nabla \times E^{0}=0$ implies the existence of a potential $\phi^{0}$ such that $E^{0}=-\nabla \phi^{0}$. Then $n_{\alpha}^{0}$ solves a classical system drift-diffusion equations:

$$
\left\{\begin{array}{l}
\partial_{t} n_{\alpha}^{0}-\operatorname{div}\left(n_{\alpha}^{0}\left(\nabla h\left(n_{\alpha}^{0}\right)+q_{\alpha} \nabla \phi^{0}\right)\right)=0 \\
-\triangle \phi^{0}=n_{i}^{0}-n_{e}^{0}
\end{array}\right.
$$

with initial condition:

$$
n_{\alpha}^{0}(0, x)=n_{\alpha, 0}
$$

Then we can get the first order companbility condition:

$$
u_{\alpha, 0}=-\nabla h\left(n_{\alpha, 0}\right)-q_{\alpha} \nabla \phi_{0}, \quad E_{0}=-\nabla \phi_{0}, \quad B_{0}=B^{0},
$$

where $\phi_{0}$ is determined by $-\triangle \phi_{0}=n_{i, 0}-n_{e, 0}$. It is similar to [6].

Secondly, let the initial data of an approximate solution $\left(n_{\alpha, \tau}, u_{\alpha, \tau}, E_{\tau}, B_{\tau}\right)(t, x)$ have an asymptotic expansion of the form:

$$
\left.\left(n_{\alpha, \tau}, u_{\alpha, \tau}, E_{\tau}, B_{\tau}\right)(t, x)\right|_{t=0}=\left(n_{\alpha, 0}, \tau u_{\alpha, 0}, E_{0}, \tau B_{0}\right)+O\left(\tau^{2}\right),
$$

where $\left(n_{\alpha, 0}, \tau u_{\alpha, 0}, E_{0}, \tau B_{0}\right)$ are the given smooth functions solutions; moreover the asymptotic expansion including initial layer correction is

$$
\begin{gathered}
\left(n_{\alpha, \tau}, u_{\alpha, \tau}, E_{\tau}, B_{\tau}\right)(t, x)=\left(n_{\alpha}^{0}, \tau u_{\alpha}^{0}, E^{0}+\tau^{2} E_{c}^{1}, \tau B^{0}\right)(t, x)+\left(\left(n_{\alpha, I}^{0}, \tau u_{\alpha, I}^{0}, E_{I}^{0}, \tau B_{I}^{0}\right)(z, x)\right. \\
\left.+\tau^{2}\left(n_{\alpha, I}^{1}, \tau u_{\alpha, I}^{1}, E_{I}^{1}, \tau B_{I}^{1}\right)\right)(z, x)+O\left(\tau^{2}\right)
\end{gathered}
$$


where $z=t / \tau^{2}$ is the fast variable, the subscript $I$ stands for the initial layer variable and $E_{c}^{1}$ is the correction term defined by:

$$
\nabla \times E_{c}^{m+1}=-\partial_{t} B^{m}, \quad \operatorname{div} E_{c}^{m+1}=0,
$$

where $m=0,1,2, \ldots$.

Obviously, $\left(n^{0}, u^{0}, E^{0}, B^{0}\right)$ satisfies the systems (2.2)-(2.5). Putting expression (2.7) into (1.11) and (1.13), we obtain $\partial_{z} n_{\alpha, I}^{0}=0, \partial_{z} E_{I}^{0}=0$ which imply

$$
n_{\alpha, I}^{0}=E_{I}^{0}=0 .
$$

Putting expression (2.7) into (1.14) and using (2.5) and (2.9), we have

$$
\partial_{z} B_{I}^{0}=0,
$$

which imply

$$
B_{I}^{0}=0 .
$$

Putting expression (2.7) into (1.12) and using (2.2), we get

$$
\partial_{z} u_{\alpha, I}^{0}+u_{\alpha, I}^{0}=0 \text {. }
$$

From (2.6)-(2.7), we have

$$
u_{\alpha}^{0}(0, x)+u_{\alpha, I}^{0}(0, x)=u_{\alpha, 0}(x),
$$

together with (2.12), we get the solution about first order initial layers for variable $u$

$$
u_{\alpha, I}^{0}(z, x)=u_{\alpha, I}^{0}(0, x) e^{-z}=\left(u_{\alpha, 0}(x)-u_{\alpha}^{0}(0, x)\right) e^{-z} .
$$

Using the similar way, we can obtain the second order initial layers

$$
\begin{aligned}
& u_{\alpha, I}^{1}(z, x)=0, \\
& \partial_{z} n_{\alpha, I}^{1}(z, x)+\operatorname{div}\left(n_{\alpha}^{0}(0, x) u_{\alpha, I}^{0}(z, x)\right)=0, \\
& \partial_{z} E_{I}^{1}(z, x)=n_{e}^{0}(0, x) u_{e, I}^{0}(z, x)-n_{i}^{0}(0, x) u_{i, I}^{0}(z, x), \\
& \partial_{z} B_{I}^{1}(z, x)+\nabla \times E_{I}^{1}(z, x)=0 .
\end{aligned}
$$

Suppose that $\left(n_{\alpha, 1}, E_{1}, B_{1}\right)$ is smooth function, and let

$$
\left(n_{\alpha, I}^{1}, E_{I}^{1}, B_{I}^{1}\right)(0, x)=\left(n_{\alpha, 1}, E_{1}, B_{1}\right)(x) .
$$

Together with (2.13), (2.14) and (2.16), we have

$$
n_{\alpha, I}^{1}(z, x)=n_{\alpha, 1}(x)-\operatorname{div}\left[n_{\alpha}^{0}(0, x)\left(u_{\alpha, 0}(x)-u_{\alpha}^{0}(0, x)\right)\right]\left(1-e^{-z}\right) .
$$

Similarly, together with (2.14), (2.17) and (2.19), we obtain

$$
E_{I}^{1}(z, x)=E_{1}+\left[n_{e}^{0}(0, x)\left(u_{e, 0}(x)-u_{e}^{0}(0, x)\right)-n_{i}^{0}(0, x)\left(u_{i, 0}(x)-u_{i}^{0}(0, x)\right)\right]\left(1-e^{-z}\right) .
$$


There we take

$$
\begin{aligned}
& E_{1}(x)=-n_{e}^{0}(0, x)\left(u_{e, 0}(x)-u_{e}^{0}(0, x)\right)-n_{i}^{0}(0, x)\left(u_{i, 0}(x)-u_{i}^{0}(0, x)\right) \\
& \operatorname{div} E_{1}(x)=n_{i, 1}(x)-n_{e, 1}(x), \quad \operatorname{div} B_{1}(x)=0 .
\end{aligned}
$$

Then we have

$$
\operatorname{div} E_{I}^{1}=n_{e, I}^{1}-n_{i, I}^{1}
$$

From a series of calculations, we get

$$
\begin{aligned}
& E_{I}^{1}(z, x)=-\left[n_{e}^{0}(0, x)\left(u_{e, 0}(x)-u_{e}^{0}(0, x)\right)-n_{i}^{0}(0, x)\left(u_{i, 0}(x)-u_{i}^{0}(0, x)\right)\right] e^{-z}, \\
& B_{I}^{1}(z, x)=B_{1}(x)+\nabla \times\left[n_{\alpha}^{0}(0, x)\left(u_{\alpha, 0}(x)-u_{\alpha}^{0}(0, x)\right)\right]\left(1-e^{-z}\right) .
\end{aligned}
$$

Then we have

$$
\operatorname{div} B_{I}^{1}=0 .
$$

According to the asymptotic expansions above, set

$$
\begin{aligned}
& n_{\alpha, \tau, I}(t, x)=n_{\alpha}^{0}(t, x)+\tau^{2} n_{\alpha, I}^{1}(z, x) \\
& u_{\alpha, \tau, I}(t, x)=\tau\left(u_{\alpha}^{0}(t, x)+u_{\alpha, I}^{0}(z, x)\right) \\
& E_{\tau, I}(t, x)=E^{0}(t, x)+\tau^{2}\left(E_{c}^{1}(t, x)+E_{I}^{1}(z, x)\right) \\
& B_{\tau, I}(t, x)=\tau B^{0}(t, x)+\tau^{3} B_{I}^{1}(z, x)
\end{aligned}
$$

Then we have

$$
\left.\left(n_{\alpha, \tau, I}, u_{\alpha, \tau, I}, E_{\tau, I}, B_{\tau, I}\right)\right|_{t=0}=\left(n_{\alpha, 0}, \tau u_{\alpha, 0}, E_{0}, \tau B_{0}\right)+\tau^{2}\left(n_{\alpha, 1}, 0, E_{1}+E_{c}^{1}(0, x), \tau B_{1}\right) .
$$

Moreover, equations (2.4), (2.8) and (2.24) imply that

$$
\operatorname{div} E_{\tau, I}=n_{i, \tau, I}-n_{e, \tau, I}
$$

and equations (2.5) and (2.27) imply that

$$
\operatorname{div} B_{\tau, I}=0 .
$$

Define the remainders $R_{n_{\alpha}}^{\tau, I}, R_{u_{\alpha}}^{\tau, I}, R_{E}^{\tau, I}$ and $R_{B}^{\tau, I}$ by

$$
\begin{aligned}
& \partial_{t} n_{\alpha, \tau, I}+\frac{1}{\tau} \operatorname{div}\left(n_{\alpha, \tau, I} u_{\alpha, \tau, I}\right)=R_{n_{\alpha}, I}^{\tau, I} \\
& \partial_{t} u_{\alpha, \tau, I}+\frac{1}{\tau}\left(u_{\alpha, \tau, I} \cdot \nabla\right) u_{\alpha, \tau, I}++\frac{1}{\tau} \nabla h\left(n_{\alpha, \tau, I}\right)=\frac{q_{\alpha}\left(E_{\tau, I}+u_{\alpha, \tau, I} \times B_{\tau, I}\right)}{\tau}-\frac{u_{\alpha, \tau, I}}{\tau^{2}}+R_{u_{\alpha}, I}^{\tau, I} \\
& \partial_{t} E_{\tau, I}-\frac{1}{\tau} \nabla \times B_{\tau, I}=\frac{n_{e, \tau, I} u_{e, \tau, I}-n_{i, \tau, I} u_{i, \tau, I}}{\tau}+R_{E}^{\tau, I}, \\
& \partial_{t} B_{\tau, I}+\frac{1}{\tau} \nabla \times E_{\tau, I}=R_{B}^{\tau, I} .
\end{aligned}
$$


Because that there is $\eta \in[0, t] \subset\left[0, T_{1}\right]$, such that

$$
n_{\alpha}^{0}(t, x)-n_{\alpha}^{0}(0, x)=t \partial_{t} n_{\alpha}^{0}(\eta, x)=\tau^{2} z \partial_{t} n_{\alpha}^{0}(\eta, x),
$$

then we have

$$
\left(n_{\alpha}^{0}(t, x)-n_{\alpha}^{0}(0, x)\right) u_{\alpha, I}^{0}=O\left(\tau^{2}\right) .
$$

After a simple calculation, we get

$$
R_{n_{\alpha}}^{\tau, I}=O\left(\tau^{2}\right), \quad R_{u_{\alpha}}^{\tau, I}=O(\tau), \quad R_{E}^{\tau, I}=O\left(\tau^{2}\right), \quad R_{B}^{\tau, I}=0 .
$$

Lemma 2.1. Let $s \geq 3$ be an integer. For given smooth data, the remainders $R_{n_{\alpha}}^{\tau, I}, R_{u_{\alpha}}^{\tau, I}, R_{E}^{\tau, I}$ and $R_{B}^{\tau, I}$ satify

$$
\sup _{0 \leq t \leq T_{1}}\left\|\left(R_{n_{\alpha}}^{\tau, I}, R_{E}^{\tau, I}\right)(t, \cdot)\right\|_{s} \leq C \tau^{2}, \quad \sup _{0 \leq t \leq T_{1}}\left\|R_{u_{\alpha}}^{\tau, I}(t, \cdot)\right\|_{s} \leq C \tau, \quad R_{B}^{\tau, I}=0,
$$

where $C>0$ is a constant independent of $\tau$.

\section{Proof of the convergence result}

In this section we prove the main convergence result from approximate periodic solution to exact solution to two-fluid Euler-Maxwell equations. Let $\left(n_{\alpha}^{\tau}, u_{\alpha}^{\tau}, E^{\tau}, B^{\tau}\right)$ be the exact solution to (1.11)-(1.14) with initial data $\left(n_{\alpha, 0}^{\tau}, u_{\alpha, 0}^{\tau}, E_{0}^{\tau}, B_{0}^{\tau}\right)$ and $\left(n_{\alpha, \tau}, u_{\alpha, \tau}, E_{\tau}, B_{\tau}\right)$ be an approximate periodic solution defined on $\left[0, T_{1}\right]$, with

$$
\left(n_{\alpha, \tau}, u_{\alpha, \tau}, E_{\tau}, B_{\tau}\right) \in C\left(\left[0, T_{1}\right], H^{s+1}(\mathbb{T})\right) \cap C^{1}\left(\left[0, T_{1}\right], H^{s}(T)\right) .
$$

By Lemma 1.1, the exact solution $\left(n_{\alpha}^{\tau}, u_{\alpha}^{\tau}, E^{\tau}, B^{\tau}\right)$ is defined in a time interval $\left[0, T_{1}^{\tau}\right]$ with $T_{1}^{\tau}>0$. Since $n_{\alpha}^{\tau} \in C\left(\left[0, T_{1}^{\tau}\right], H^{s}(\mathbb{T})\right.$ and the embedding from $H^{s}(\mathbb{T})$ to $C(\mathbb{T})$ is continuous, we have $n_{\alpha}^{\tau} \in C\left(\left[0, T_{1}^{\tau}\right] \times \mathbb{T}\right)$. From (1.17) and (1.18) and assumption $n_{\alpha, 0}^{\tau} \geq k>0$, we deduce that there exist $T_{2}^{\tau} \in\left(0, T_{1}^{\tau}\right]$ and a constant $C_{0}>0$, independent of $\tau$, such that

$$
\frac{k}{2} \leq n_{\alpha}^{\tau} \leq C_{0}, \quad \forall(t, x) \in\left[0, T_{2}^{\tau}\right] \times \mathbb{T} .
$$

Similarly, the function $t \mapsto\left\|\left(n_{\alpha}^{\tau}(t, \cdot), u_{\alpha}^{\tau}(t, \cdot), E^{\tau}(t, \cdot), B^{\tau}(t, \cdot)\right)\right\|_{s}$ is continuous in $C\left(\left[0, T_{2}^{\tau}\right]\right)$. From (1.17), the sequence $\left(\left\|\left(n_{\alpha}^{\tau}(0, \cdot), u_{\alpha}^{\tau}(0, \cdot), E^{\tau}(0, \cdot), B^{\tau}(0, \cdot)\right)\right\|_{s}\right)_{\tau>0}$ is bounded. Then there exist $T_{3}^{\tau} \in\left(0, T_{2}^{\tau}\right]$ and a constant, still denoted by $C_{0}$, such that

$$
\left\|\left(n_{\alpha}^{\tau}(t, \cdot), u_{\alpha}^{\tau}(t, \cdot), E^{\tau}(t, \cdot), B^{\tau}(t, \cdot)\right)\right\|_{s} \leq C_{0}, \quad \forall t \in\left(0, T_{3}^{\tau}\right] .
$$

Then we define $T^{\tau}=\min \left(T_{1}, T_{3}^{\tau}\right)>0$, such that the exact solution and the approximate are both defined on $\left[0, T^{\tau}\right]$, and the exact solution satisfy:

$$
\frac{k}{2} \leq n_{\alpha}^{\tau} \leq C, \quad\left\|\left(n_{\alpha}^{\tau}, u_{\alpha}^{\tau}, E^{\tau}, B^{\tau}\right)\right\|_{s} \leq C,
$$


where $C>0$ is a constant independent of $\tau$. Obviously it is valid from the paper [6].

Let

$$
\left(N_{\alpha}^{\tau}, U_{\alpha}^{\tau}, F^{\tau}, G^{\tau}\right)=\left(n_{\alpha}^{\tau}-n_{\alpha, \tau}, u_{\alpha}^{\tau}-u_{\alpha, \tau}, E^{\tau}-E_{\tau}, B^{\tau}-B_{\tau}\right),
$$

obviously the error function $\left(N_{\alpha}^{\tau}, U_{\alpha}^{\tau}, F^{\tau}, G^{\tau}\right)$ satisfies:

$$
\begin{aligned}
& \partial_{t} N_{\alpha}^{\tau}+\frac{1}{\tau}\left(\left(U_{\alpha}^{\tau}+u_{\alpha, \tau}\right) \cdot \nabla\right) N_{\alpha}^{\tau}+\frac{1}{\tau}\left(N_{\alpha}^{\tau}+n_{\alpha, \tau}\right) \operatorname{div} U_{\alpha}^{\tau} \\
= & -\frac{1}{\tau}\left(N_{\alpha}^{\tau} \operatorname{div} u_{\alpha, \tau}+\left(U_{\alpha}^{\tau} \cdot \nabla\right) u_{\alpha, \tau}\right)-R_{n_{\alpha^{\prime}}}^{\tau} \\
& \partial_{t} U_{\alpha}^{\tau}+\frac{1}{\tau}\left(\left(U_{\alpha}^{\tau}+u_{\alpha, \tau}\right) \cdot \nabla\right) U_{\alpha}^{\tau}+\frac{1}{\tau} h^{\prime}\left(N_{\alpha}^{\tau}+n_{\alpha, \tau}\right) \nabla N_{\alpha}^{\tau} \\
= & -\frac{1}{\tau}\left[\left(U_{\alpha}^{\tau} \cdot \nabla\right) u_{\alpha, \tau}+\left(h^{\prime}\left(N_{\alpha}^{\tau}+n_{\alpha, \tau}\right)+h^{\prime}\left(n_{\alpha, \tau}\right)\right)\right] \\
& \quad-\frac{1}{\tau^{2}} U_{\alpha}^{\tau}-\frac{1}{\tau} q_{\alpha}\left[F^{\tau}+\left(U_{\alpha}^{\tau}+u_{\alpha, \tau}\right) \times G^{\tau}+U_{\alpha}^{\tau} \times B_{\tau}\right]-R_{u_{\alpha^{\prime}}}^{\tau}, \\
& \partial_{t} F^{\tau}-\frac{1}{\tau} \nabla \times G^{\tau} \\
= & \frac{1}{\tau}\left[\left(N_{e}^{\tau} U_{e}^{\tau}-N_{i}^{\tau} U_{i}^{\tau}\right)+\left(n_{e, \tau} U_{e}^{\tau}-n_{i, \tau} U_{i}^{\tau}\right)+\left(N_{e}^{\tau} u_{e, \tau}-N_{i}^{\tau} u_{i, \tau}\right)\right]-R_{E}^{\tau}, \\
& \partial_{t} G^{\tau}+\frac{1}{\tau} \nabla \times F^{\tau}=0, \quad \operatorname{div} F^{\tau}=N_{i}^{\tau}-N_{e}^{\tau} .
\end{aligned}
$$

Set

$$
\begin{aligned}
& W_{I}^{\tau}=\left(\begin{array}{c}
N_{\alpha}^{\tau} \\
U_{\alpha}^{\tau}
\end{array}\right), \quad W_{I I}^{\tau}=\left(\begin{array}{c}
F^{\tau} \\
G^{\tau}
\end{array}\right), \quad W^{\tau}=\left(\begin{array}{c}
W_{I}^{\tau} \\
W_{I I}^{\tau}
\end{array}\right)=\left(\begin{array}{c}
N_{\alpha}^{\tau} \\
U_{\alpha}^{\tau} \\
F^{\tau} \\
G^{\tau}
\end{array}\right), \\
& H_{1}\left(W_{I}^{\tau}\right)=\left(\begin{array}{c}
-\left(U_{\alpha}^{\tau} \cdot \nabla\right) n_{\alpha, \tau}-N_{\alpha}^{\tau} \operatorname{div} u_{\alpha, \tau} \\
-\left(U_{\alpha}^{\tau} \cdot \nabla\right) u_{\alpha, \tau}-\left(h_{\alpha}^{\prime}\left(N_{\alpha}^{\tau}+n_{\alpha, \tau}\right)-h_{\alpha}^{\prime}\left(n_{\alpha, \tau}\right)\right) \nabla n_{\alpha, \tau}
\end{array}\right), \\
& H_{2}\left(W_{I}^{\tau}\right)=\left(\begin{array}{c}
0 \\
-U_{\alpha}^{\tau}
\end{array}\right), H_{3}\left(W^{\tau}\right)=\left(\begin{array}{c}
q_{\alpha}\left[F^{\tau}+\left(U_{\alpha}^{\tau}+u_{\alpha, \tau}\right) \times G^{\tau}+U_{\alpha}^{\tau} \times B_{\tau}\right]
\end{array}\right), \\
& R^{\tau}=\left(\begin{array}{c}
R_{n_{\alpha}}^{\tau} \\
R_{u_{\alpha}}^{\tau}
\end{array}\right), A_{j}^{I}\left(n_{\alpha}^{\tau}, u_{\alpha}^{\tau}\right)=\left(\begin{array}{cc}
u_{\alpha, j}^{\tau} & n_{\alpha}^{\tau} e_{i}^{t} \\
h_{\alpha}^{\prime}\left(n_{\alpha}^{\tau}\right) e_{j} & u_{\alpha, j}^{\tau} I_{3}
\end{array}\right), \quad j=1,2,3,
\end{aligned}
$$

where $\alpha=e, i,\left(e_{1}, e_{2}, e_{3}\right)$ is the canonical basis of $R^{3}, y_{j}$ denotes the $i$ th component of $y \in R^{3}$ and $I_{3}$ is the $3 \times 3$ unit matrix. Then systems (3.3) and (3.4) for unknown $W_{I}^{\tau}$ can be rewritten as

$$
\partial_{t} W_{I}^{\tau}+\frac{1}{\tau} \sum_{i=1}^{3} A_{i}^{I}\left(n_{\alpha}^{\tau}, u_{\alpha}^{\tau}\right) \partial_{x_{i}} W_{I}^{\tau}=\frac{1}{\tau}\left(H_{1}\left(W_{I}^{\tau}\right)+H_{3}\left(W^{\tau}\right)\right)+\frac{1}{\tau^{2}} H_{2}\left(W_{I}^{\tau}\right)-R^{\tau} .
$$


It is symmetrizable hyperbolic with symmetrizer

$$
A_{0}^{I}\left(n_{\alpha}^{\tau}\right)=\left(\begin{array}{cc}
\left(n_{\alpha}^{\tau}\right)^{-1} & 0 \\
0 & \left(h_{\alpha}^{\prime}\left(n_{\alpha}^{\tau}\right)\right)^{-1} I_{3}
\end{array}\right),
$$

which is a positive definite matrix when $0<\frac{k}{2} \leq n_{\alpha}^{\tau}=N_{\alpha}^{\tau}+n_{\alpha, \tau} \leq C$, where $\alpha=e, i$. Moreover

$$
\widetilde{A}_{i}^{I}\left(n_{\alpha}^{\tau}, u_{\alpha}^{\tau}\right)=A_{0}^{I}\left(n_{\alpha}^{\tau}\right) A_{i}^{I}\left(n_{\alpha}^{\tau}, u_{\alpha}^{\tau}\right)=u_{\alpha, j}^{\tau} A_{0}^{I}\left(n_{\alpha}^{\tau}\right)+D_{i}
$$

is symmetric for all $1 \leq j \leq 3$, where each $D_{j}$ is a constant matrix

$$
D_{j}=\left(\begin{array}{cc}
0 & e_{j}^{t} \\
e_{j} & 0
\end{array}\right) .
$$

In order to prove Theorem 1.1, it suffices to establish uniform estimates of $W^{\tau}$ with respect to $\tau$. In what follows, we denote by $C>0$ various constants independent of $\tau$ and for $\beta \in N^{3},\left(W_{I, \beta}^{\tau}, W_{I I, \beta}^{\tau}\right)=\partial_{x}^{\beta}\left(W_{I}^{\tau}, W_{I I}^{\tau}\right)$, etc. The main estimates are contained in the following two lemmas for $W_{I}^{\tau}$ and $W_{I I}^{\tau}$, respectively. We first consider the estimate for $W_{I}^{\tau}$.

Lemma 3.1. Under the assumptions of Theorem 1.1, for all $t \in\left(0, T^{\tau}\right]$, as $\tau \rightarrow 0$ we have

$$
\left\|W_{I}^{\tau}(t)\right\|_{s}^{2}+\frac{1}{\tau^{2}} \int_{0}^{t}\left\|U_{\alpha}^{\tau}(t)\right\|_{s}^{2} \mathrm{~d} \xi \leq C \int_{0}^{t}\left(\left\|W^{\tau}(\xi)\right\|_{s}^{2}+\left\|W^{\tau}(\xi)\right\|_{s}^{4}\right) \mathrm{d} \xi+C \tau^{4} .
$$

Proof. Differentiating equation (3.7) with respect to $x$ yields and then multiplying by $A_{0}^{I}\left(n_{\alpha}^{\tau}\right)$ and taking the inner product of the resulting equation with $W_{I \beta}^{\tau}$, we obtain

$$
\begin{aligned}
& \frac{\mathrm{d}}{\mathrm{d} t}\left(A_{0}^{I}\left(n_{\alpha}^{\tau}\right) W_{I, \beta}^{\tau}, W_{I, \beta}^{\tau}\right)-\frac{2}{\tau}\left(A_{0}^{I}\left(n_{\alpha}^{\tau}\right) \partial_{x}^{\beta} H_{2}\left(W_{I}^{\tau}\right), W_{I, \beta}^{\tau}\right) \\
=\frac{2}{\tau}(( & \left.\left.A_{0}^{I}\left(n_{\alpha}^{\tau}\right)\left[\partial_{x}^{\beta} H_{1}\left(W_{I}^{\tau}\right)+\partial_{x}^{\beta} H_{3}\left(W^{\tau}\right)\right] \cdot W_{I, \beta}^{\tau}\right), W_{I, \beta}^{\tau}\right) \\
& \quad+\frac{2}{\tau}\left(J_{\beta}^{\tau}, W_{I, \beta}^{\tau}\right)+\left(\operatorname{div} A_{\tau}^{I}\left(n_{\alpha}^{\tau}, u_{\alpha}^{\tau}\right) W_{I, \beta}^{\tau}, W_{I, \beta}^{\tau}\right)-2\left(A_{0}^{I}\left(n_{\alpha}^{\tau}\right) \partial_{x}^{\beta} R^{\tau}, W_{I, \beta}^{\tau}\right),
\end{aligned}
$$

where

$$
\begin{aligned}
& J_{\beta}^{\tau}=-\sum_{j=1}^{3} A_{0}^{I}\left(n_{\alpha}^{\tau}\right)\left[\partial_{x}^{\beta}\left(A_{j}^{I}\left(n_{\alpha}^{\tau}, u_{\alpha}^{\tau}\right) \partial_{x_{i}} W_{I}^{\tau}\right)-A_{j}^{I}\left(n_{\alpha}^{\tau}, u_{\alpha}^{\tau}\right) \partial_{x}^{\beta} \partial_{x_{i}} W_{I}^{\tau}\right], \\
& \operatorname{div} A_{\tau}^{I}\left(n_{\alpha}^{\tau}, u_{\alpha}^{\tau}=\partial_{t} A_{0}^{I}\left(n_{\alpha}^{\tau}+\frac{1}{\tau} \sum_{j=1}^{3} \partial_{x_{j}} \widetilde{A}_{i}^{I}\left(n_{\alpha}^{\tau}, u_{\alpha}^{\tau}\right) .\right.\right.
\end{aligned}
$$

From estimating each term of equation (3.10), for $\forall|\beta| \leq s$, we have

$$
\frac{\mathrm{d}}{\mathrm{d} t}\left(A_{0}^{I}\left(n_{\alpha}^{\tau}\right) W_{I, \beta}^{\tau}, W_{I, \beta}^{\tau}\right)+\frac{k}{\tau^{2}} \leq \frac{C_{\varepsilon}}{\tau^{2}}\left\|U_{\alpha}^{\tau}\right\|_{s}^{2}+C_{\varepsilon}\left(\left\|W^{\tau}\right\|_{s}^{2}+\left\|W^{\tau}\right\|_{s}^{2}\right)+C_{\varepsilon} \tau^{4} .
$$

Integrating this equation over $(0, t)$ with $t \in\left(0, T^{\tau}\right)$ and summing up over all $|\beta| \leq s$, taking $\varepsilon \geq 0$ sufficiently small that the term including $\frac{C_{\varepsilon}}{\tau^{2}}\left\|U_{\alpha}^{\tau}\right\|_{s}^{2}$ can be controlled by the left-hand side, together with condition (1.18) for the initial data, we get (3.9). 
The estimate for $W_{I}^{\tau}$ is similar to the paper [6], so we give the simple steps. Now we establish the estimate for $W_{I I}^{\tau}$, it will be complicated because there exist both electron and ion.

Lemma 3.2. Under the assumptions of Theorem 1.1, for all $t \in\left(0, T^{\tau}\right]$, as $\tau \rightarrow 0$ we have

$$
\left\|W_{I I}^{\tau}(t)\right\|_{s}^{2} \leq \int_{0}^{t}\left(\frac{1}{\tau^{2}}\left\|U_{\alpha}^{\tau}(t)\right\|_{s}^{2}+\left\|W^{\tau}(\xi)\right\|_{s}^{2}+\left\|W^{\tau}(\xi)\right\|_{s}^{4}\right) \mathrm{d} \xi+C \tau^{4}
$$

Proof. For a multi-index $\beta \in N^{3}$ with $|\beta| \leq s$, differentiating the equations (3.5) and (3.6) with respect to $x$, we have

$$
\begin{aligned}
\partial_{t} F_{\beta}^{\tau}-\frac{1}{\tau} \nabla \times G_{\beta}^{\tau}=\frac{1}{\tau} \partial_{x}^{\beta} & {\left[\left(N_{e}^{\tau} U_{e}^{\tau}-N_{i}^{\tau} U_{i}^{\tau}\right)+\left(n_{e, \tau} U_{e}^{\tau}-n_{i, \tau} U_{i}^{\tau}\right)\right.} \\
& \left.+\left(N_{e}^{\tau} u_{e, \tau}-N_{i}^{\tau} u_{i, \tau}\right)\right]-\partial_{x}^{\beta} R_{E}^{\tau},
\end{aligned}
$$

and

$$
\partial_{t} G_{\beta}^{\tau}+\frac{1}{\tau} \nabla \times F_{\beta}^{\tau}=0
$$

Taking the inner product of equation (3.15) with $F_{\beta}^{\tau}$ and taking the inner product of equation (3.16) with $G_{\beta}^{\tau}$, then we have

$$
\begin{aligned}
\int_{\mathbb{T}} \frac{1}{2}\left[\partial_{t}\left(F_{\beta}^{\tau}\right)^{2}+\partial_{t}\left(G_{\beta}^{\tau}\right)^{2}\right] \mathrm{d} x=\frac{1}{\tau} \int_{\mathbb{T}} \partial_{x}^{\beta}\left[\left(N_{e}^{\tau} U_{e}^{\tau}-N_{i}^{\tau} U_{i}^{\tau}\right)+\left(n_{e, \tau} U_{e}^{\tau}-n_{i, \tau} U_{i}^{\tau}\right)\right. \\
\left.+\left(N_{e}^{\tau} u_{e, \tau}-N_{i}^{\tau} u_{i, \tau}\right)\right] \cdot F_{\beta}^{\tau}-\partial_{x}^{\beta} R_{E}^{\tau} \cdot F_{\beta}^{\tau} \mathrm{d} x
\end{aligned}
$$

there because

$$
\int_{\mathbb{T}}\left(\frac{1}{\tau} \nabla \times G_{\beta}^{\tau} \cdot F_{\beta}^{\tau}-\frac{1}{\tau} \nabla \times F_{\beta}^{\tau} \cdot G_{\beta}^{\tau}\right) \mathrm{d} x=\frac{1}{\tau} \int_{\mathbb{T}} \operatorname{div}\left(F_{\beta}^{\tau} \times G_{\beta}^{\tau}\right) \mathrm{d} x=0 .
$$

Then we have

$$
\begin{aligned}
& \frac{\mathrm{d}}{\mathrm{d} t}\left\|W_{I I \beta}^{\tau}(t)\right\|^{2} \leq \int_{\mathbb{T}} \mid \partial_{x}^{\beta}\left[\left(N_{e}^{\tau} U_{e}^{\tau}-N_{i}^{\tau} U_{i}^{\tau}\right)+\left(n_{e, \tau} U_{e}^{\tau}-n_{i, \tau} U_{i}^{\tau}\right)\right. \\
&\left.+\left(N_{e}^{\tau} u_{e, \tau}-N_{i}^{\tau} u_{i, \tau}\right)\right] \cdot F_{\beta}^{\tau}\left|\mathrm{d} x+\int_{\mathbb{T}}\right| \partial_{x}^{\beta} R_{E}^{\tau} \cdot F_{\beta}^{\tau} \mid \mathrm{d} x .
\end{aligned}
$$

Using energy estimate, (1.21) and Lemma 2.1, we obtain

$$
\frac{\mathrm{d}}{\mathrm{d} t}\left\|W_{I I \beta}^{\tau}(t)\right\|^{2} \leq \frac{1}{2 \tau^{2}}\left\|U_{\alpha}^{\tau}(t)\right\|_{s}^{2}+C\left\|W^{\tau}(\xi)\right\|_{s}^{2}+C\left\|W^{\tau}(\xi)\right\|_{s}^{4}+C \tau^{4} .
$$

Integrating (3.20) over $(0, t)$, summing up over $\beta$ satisfying $|\beta| \leq s$ and using (1.18), we obtain the lemma. 
Proof of Theorem 1.1. Let $\tau \rightarrow 0$ and $\varepsilon>0$ be sufficiently small. By Lemma 3.1 and Lemma 3.2, for $t \in\left(0, T^{\tau}\right]$ we have

$$
\left\|W^{\tau}(t)\right\|_{s}^{2}+\frac{1}{\tau^{2}} \int_{0}^{t}\left\|U_{\alpha}^{\tau}(t)\right\|_{s}^{2} \mathrm{~d} \xi \leq C \int_{0}^{t}\left(\left\|W^{\tau}(\xi)\right\|_{s}^{2}+\left\|W^{\tau}(\xi)\right\|_{s}^{4}\right) \mathrm{d} \xi+C \tau^{4} .
$$

Let

$$
y(t)=C \int_{0}^{t}\left\|W^{\tau}(\xi)\right\|_{s}^{2}+\left\|W^{\tau}(\xi)\right\|_{s}^{4} \mathrm{~d} \xi+C \tau^{4} .
$$

Then it follows from (3.21) that

$$
\begin{aligned}
& \left\|W^{\tau}(t)\right\|_{s}^{2} \leq y(t), \quad \frac{1}{\tau^{2}} \int_{0}^{t}\left\|U_{\alpha}^{\tau}(t)\right\|_{s}^{2} \mathrm{~d} \xi \leq y(t), \quad \forall t \in\left(0, T^{\tau}\right], \\
& y^{\prime}(t)=C\left(\left\|W^{\tau}(\xi)\right\|_{s}^{2}+\left\|W^{\tau}(\xi)\right\|_{s}^{4}\right) \leq C\left(y(t)+y^{2}(t)\right),
\end{aligned}
$$

with

$$
y(0)=C \tau^{4}
$$

From Gronwall inequality, we get

$$
y(t) \leq C \tau^{4} e^{C t} \leq C \tau^{4} e^{C T_{1}}, \quad \forall t \in\left[0, T^{\tau}\right] .
$$

Therefore, from (3.23) we obtain

$$
\left\|W^{\tau}(t)\right\|_{s} \leq \sqrt{y(t)} \leq C \tau^{2}, \quad \int_{0}^{t}\left\|U_{\alpha}^{\tau}(\xi)\right\|_{s}^{2} \mathrm{~d} \xi \leq \tau^{2} y(t) \leq C \tau^{6} .
$$

By a standard argument on the time extension of smooth solutions, we obtain $T_{3}^{\tau} \geq T_{1}$, i.e. $T^{\tau}=T_{1}$. This finishes the proof of Theorem 1.1.

\section{Acknowledgement}

The authors thank the referee for various comments which allow to improve the presentation of the paper.

\section{References}

[1] Chen G.Q., Jerome J.W. and Wang D.H., Compressible Euler-Maxwell equations. Transport Theory and Statist. Phys. 29 (2000), 311-331.

[2] Peng Y.J., Wang S., Convergence of compressible Euler-Maxwell equations to compressible Euler-Poisson equations. Chinese Ann. Math. Ser. B 28 (2007), 583-602.

[3] Peng Y.J., Wang S., Rigorous derivation of incompressible e-MHD equations from compressible Euler-Maxwell equations. SIAM J. Math. Anal. 40 (2008), 540-565.

[4] Peng Y.J., Wang S. and Gu Q. L., Relaxation limit and global existence of smooth solutions of compressible Euler-Maxwell equations. SIAM J. Math. Anal. 43 (2011), 944-970. 
[5] Yang J.W., Wang S. and Zhao J., The relaxation limit in the compressible Euler-Maxwell equations. Nonlinear Anal. 74 (2011), 7005-7011.

[6] Hajjej M.L., Peng Y.J., Initial layers and zero-relaxation limits of Euler-Maxwell equations. J. Differential Equations 52 (2012), 1441-1465.

[7] Admas R.A., Sobolev Spaces. Academic Press, American, 1975.

[8] Wang S., Sobolev Space and Introduction to Partial Differential Equations. Science Press, China, 2009.

[9] Kao T., The cauchy problem for quasi-linear symmetric hyperbolic systems. Arch. Ration. Mech. Anal. 58(1975), 181-205.

[10] Majda A., Compressible Fluid Flow and Systems of Conservation Laws in Several Space Variable. Springer-Verlag, NewYork, 1984. 